\title{
Competencias digitales e innovación educativa en la conversión de curso presencial en curso virtual durante la pandemia de COVID-19
}

\author{
Ana María Martín-Cuadrado ${ }^{1}$ \\ Fernando José Spanhol ${ }^{2}$ \\ Lourdes Pérez Sánchez ${ }^{3}$ \\ Mabel Alvarez ${ }^{4}$ \\ Rangel Machado Simon ${ }^{5}$
}

\section{RESUMEN}

Este trabajo pretende dar cuenta de la experiencia sobre la participación en un curso virtual de Capacitación de Formadores en la Competencia Digital de Comunicación y Colaboración, aplicado a funcionarios públicos de América Latina y el Caribe (ALC), originalmente preparado para ser presentado de manera híbrida y posteriormente adaptado para ser totalmente a distancia, debido a la pandemia ocasionada por la COVID-19. El curso se enmarca en el Programa de Capacitación de Formadores en Competencias Digitales coordinado por la Universidad de Educación a Distancia (UNED) en asociación con la OEI-Panamá y el Convenio Andrés Bello. En este Programa participan: la Universidad Nacional de la Patagonia San Juan Bosco (Argentina) y la Universidad Federal de Santa Catarina (Brasil). El Programa forma parte del Plan INTERCOONECTA y se encuentra financiado por la Agencia Española de Cooperación Internacional para el Desarrollo (AECID). Para adaptar la formación a la virtualidad esta experiencia utilizó como metodología el Aprendizaje Basado en Problemas (ABP) con la mediación de profesores y tutores en un entorno virtual de aprendizaje (EVA) desarrollado en Moodle, debidamente estructurado para apoyar las acciones de cursos de esta naturaleza. La experiencia lograda en el curso indica que la educación debe tener una mirada diferente en la aplicación de modelos de este tipo, la transmisión de los conocimientos obtenidos a otros compañeros de la institución, pudiendo así maximizar y desarrollar habilidades de comunicación y colaboración digital de todos los involucrados.

Palabras-clave: Competencias digitales. Entornos virtuales de aprendizaje. Aprendizaje basado en problemas. Comunicación y colaboración.

\footnotetext{
${ }^{1}$ amartin@edu.uned.es - Universidad Nacional de Educacion a Distancia (UNED)

2 profspanhol@gmail.com - Universidade Federal de Santa Catarina (UFSC)

3 Iperezsanchez@edu.uned.es - Universidad Nacional de Educación a Distancia (UNED)

${ }^{4}$ mabelalvarez10@gmail.com - Universidad Nacional de la Patagonia San Juan Bosco (UNSJB)

${ }^{5}$ rangel.simon@gmail.com - Universidade Federal de Santa Catarina (UFSC)
} 


\section{Digital competencies for educational innovation in the conversion from face-to-face to virtual course during the pandemic of COVID 19}

\section{ABSTRACT}

This paper aims to give an account of the experience of participating in a virtual Training of Trainers course in Digital Communication and Collaboration Competence, applied to public officials from Latin America and the Caribbean (LAC), originally prepared to be presented in a hybrid way and later adapted to be totally remote, due to the pandemic caused by COVID-19. The course is part of the Training Program for Trainers in Digital Competences coordinated by the Distance Education University (UNED) in association with the OEI-Panama and the Andrés Bello Agreement. Participating in this Program are: the San Juan Bosco National University of Patagonia (Argentina) and the Federal University of Santa Catarina (Brazil). The Program is part of the INTERCOONECTA Plan and is funded by the Spanish Agency for International Development Cooperation (AECID). To adapt the training to virtuality, this experience used Problem-Based Learning (PBL) as a methodology with the mediation of teachers and tutors in a virtual learning environment (VLE) developed in Moodle, duly structured to support the actions of courses of this nature. That said, the experience gained in the course indicates that education should take a different look at the application of models of this type. This is because it is one of the conditions for the participants in this course, the transmission of the knowledge obtained to other colleagues of the institution, thus being able to maximize and develop communication skills and digital collaboration of all those involved.

Keywords: Digital competence. Virtual Learning Environments. Problem-based learning. Communication and collaboration. 
En 2020 el mundo comienza a sufrir los impactos de lo que en el futuro sería declarado pandemia COVID-19 por la Organización Mundial de la Salud (OMS). Los países crearon diferentes restricciones para reducir la circulación del virus, pero en general se cerraron las fronteras, se suspendieron las clases y se llevaron a casa las actividades presenciales, realizándolas mediante el uso de las Tecnologías de la Información y la Comunicación (TIC).

A partir de una transición gradual de los medios analógicos a los digitales, muchas personas se han visto abocadas a este nuevo modelo de aprendizaje y enseñanza, con pocas competencias sobre cómo y cuándo utilizar determinadas tecnologías.

La COVID-19 nos ha puesto a prueba, no sólo de nuestras capacidades sociales y sanitarias, sino también de nuestras habilidades para abordar la educación a través de las TIC.

En este sentido, el presente trabajo pretende dar cuenta de la experiencia de un curso sobre competencias digitales aplicado a funcionarios públicos de América Latina y el Caribe, originalmente preparado para ser presentado de manera híbrida y posteriormente adaptado para ser totalmente a distancia, debido a la pandemia de coronavirus.

El curso de Formación de Formadores en Competencias Digitales basado en DigComp (Lucas \& Moreira, 2017) se enmarca en el Programa de Capacitación de Formadores en Competencias Digitales coordinado por la Universidad de Educación a Distancia (UNED) en asociación con la OEI-Panamá y el Convenio Andrés Bello. En este Programa participan: la Universidad Nacional de la Patagonia San Juan Bosco (Argentina) y la Universidad Federal de Santa Catarina (Brasil). El Programa forma parte del Plan INTERCOONECTA y se encuentra financiado por la Agencia Española de Cooperación Internacional para el Desarrollo (AECID).

La experiencia utilizó la metodología del Aprendizaje Basado en Problemas (ABP) y fue realizada a distancia, a través del entorno virtual de aprendizaje Moodle de la AECID.

\section{LA PANDEMIA Y LA EDUCACIÓN}

Debido al proceso mencionado anteriormente, el Programa de Formador de Formadores en Competencias Digitales, que utilizaría la modalidad híbrida de enseñanza, teniendo clases presenciales en los países de América Latina y el Caribe y luego continuando a través de una plataforma de enseñanza en línea, se modificó para ejecutarse totalmente a distancia, utilizando las TIC para este proceso. El cual se basó en los principios europeos de actuación para la educación digital (Comisión Europea, 2020), que de forma resumida comprenden:

a) una educación digital inclusiva y de alta calidad, que respete la protección de los datos personales y los valores éticos, debe ser un objetivo estratégico de todos los organismos y agencias activos en la educación y la formación;

b) transformar la educación para la era digital es una tarea que le corresponde al conjunto de la sociedad; 
c) una inversión adecuada en conectividad, equipos, capacidades y destrezas organizativas debería garantizar que todo el mundo tenga acceso a la educación digital;

d) la educación digital debería desempeñar un papel fundamental a la hora de potenciar la igualdad y mejorar la inclusión;

e) la competencia digital debería ser una capacidad básica para todos los educadores y el personal de formación;

f) los líderes del sector educativo desempeñan un papel clave en la educación digital;

g) la alfabetización digital es fundamental para la vida en un mundo digitalizado;

h) las capacidades digitales básicas deberían formar parte de las capacidades básicas transferibles que todas las personas deberían tener para poder desarrollarse a nivel personal, participar en el conjunto de la sociedad como ciudadanos activos, utilizar los servicios públicos y ejercer sus derechos básicos;

i) para apoyar la competitividad, necesitamos que las personas cuenten con las capacidades digitales más avanzadas;

j) contenido educativo de alta calidad que impulse la pertinencia, la calidad y la inclusividad de la educación y la formación europeas a todos los niveles.

Elcurso de Capacitación de Formadores en la Competencia Digital de Comunicación y Colaboración se diseñó totalmente para impartirlo de forma virtual, teniendo en cuenta los principios enumerados anteriormente. Por ello, el uso de las TIC fue esencial en la construcción del conocimiento mediante la interacción de participantes con el equipo docente y entre participantes. En este proceso, aunque la mayoría de ellos ya estaban familiarizados con el uso de las TIC, se encontraron varios retos señalados por los estudiantes, como los problemas de conexión, las diferentes infraestructuras en los distintos países a los que se dirigía la capacitación y los diferentes niveles de compromiso en los procesos educativos.

Aunque, para muchos, el uso de las TIC ya de alguna manera formaba parte de la rutina de trabajo, su utilización para sustituir las reuniones presenciales generó impactos diferentes y dio lugar a una mayor atención a problemas que antes se dejaban de lado.

El uso de una metodología activa, como el Aprendizaje Basado en Problemas (ABP), vino a satisfacer los requisitos de regionalización de las actividades propuestas. Esto posibilitó que los estudiantes muestren los problemas que les interesaba resolver con el uso de las TIC, dando un papel más activo a los participantes, fortaleciendo la interacción entre estudiantes y tutores y entre participantes de diferentes países. Este papel es fundamental para una mayor integración entre estudiantes y educadores y, en el caso de este curso, para compartir conocimientos entre diferentes países.

Al tratar la virtualización de las clases, Moreira \& Schlemmer (2020, p. 9), hablan de la enseñanza a distancia:

[...] La enseñanza presencial física (los mismos cursos, planes de estudio, metodologías y prácticas docentes) se traslada a los medios digitales en red. El proceso se centra en los contenidos, que son impartidos por el mismo profesor que la clase presencial. Aunque haya una distancia geográfica, se privilegia el compartir el mismo tiempo, es decir, la 
clase se desarrolla en un tiempo sincrónico, siguiendo los principios de la enseñanza presencial. La comunicación es predominantemente bidireccional, del tipo uno a muchos, en la que el profesor es el protagonista de una videoaula o realiza una lección expositiva a través de sistemas de conferencia web. De este modo, la presencia física del profesor y del alumno en el espacio geográfico del aula se sustituye por una presencia digital en un aula digital. En la webconferencia o el aula a distancia, la atención se centra en la información y en las formas de transmitirla.

Esta acción se alía con las estructuras de educación a distancia (EaD), en las que hay que pensar en estrategias educativas para mejorar proceso de enseñanza.

En este sentido, cabe destacar también la capacidad de las Tecnologías de la Información y la Comunicación para conectar a diferentes actores a través de espacios digitales construidos con el fin de intercambiar información y construir conocimiento. Los Entornos Virtuales de Enseñanza y Aprendizaje son, según Catapan (2002), un sistema que proporciona diversas herramientas de comunicación e interacción. En este enfoque, tienen el propósito de promover el aprendizaje no desconectado de un proceso de enseñanza que es sistemático, organizado, intencional y de carácter formal.

El formato de educación en línea, por su parte, es un enfoque didáctico-pedagógico (Santos, 2009; Pimentel \& Carvalho, 2020) que no puede dejar de mencionarse, ya que es ampliamente utilizado a lo largo del curso. Según Santos (2009), la educación en línea es uno de los procesos resultantes de la cibercultura y no una evolución de la EaD y aporta el ejercicio de la autoría, el fomento de la autonomía, la comunicación colaborativa en red, la interactividad, el diálogo, según los principios en los que se basa. A efectos de lograr un máximo provecho es necesario que el usuario del sistema tenga la competencia necesaria para ello.

\section{3}

\section{COMPETENCIAS DIGITALES}

La competencia, según Setzer (1999), es la capacidad de producir algo, en un área de conocimiento determinada. Por tanto, es la capacidad que tiene cada individuo para poner en práctica los conocimientos obtenidos, ya sea algo tangible o intangible.

Cabe destacar que la trayectoria para la creación de conocimiento se origina en los datos que, al ser contextualizados, generan información. Este conjunto de datos con relaciones y dotados de relevancia crean significado. Sólo al utilizar la capacidad cognitiva para procesar la información que, según Uriarte Junior (2008), es donde el ser humano adquiere el conocimiento y cuando es capaz de usarlo y transmitirlo, lo transforma en competencia.

Se puede ver que la diferencia entre conocimiento y competencia radica en su uso. Cuando sólo se adquiere, se tiene conocimiento. Pero es al poner en práctica esta adquisición cuando se demuestra la capacidad de utilizarla; es en otras palabras, la competencia.

Al llevar este concepto a los entornos digitales, vemos que las competencias digitales son la capacidad de realizar actividades en estos espacios, para recopilar y crear acciones educativas directamente dirigidas a ello. En el texto de DigComp, según Lucas y Moreira (2017) hay una recopilación y segmentación de las principales áreas de competencia. 
Antes, sólo se necesitaban acciones analógicas, pero se ha cambiado a pensamientos donde lo digital debe estar presente para que ciertas acciones se ejecuten hasta el final. Esto requiere tener presente que no se trata de saber, sino de saber hacer, que es donde se asigna la competencia.

Pero, aunque esta transición de la información analógica a la digital parece intuitiva, con el impacto de la inserción de la tecnología en el mercado laboral no significó necesariamente la transformación del trabajo analógico en digital. El saber hacer, es decir, la formación de las personas para insertarse adecuadamente en una sociedad digital es fundamental, ya que la necesidad de formar a las personas para ser ciudadanos del siglo XXI es una preocupación a nivel institucional e internacional.

Organizaciones internacionales como la Organización de las Naciones Unidas para la Educación, la Ciencia y la Cultura (UNESCO, 2017a; 2017b), la OCDE (2019) y la Comisión Europea (2006; Vuorikari et al., 2016) llevan algunos años trabajando, y siguen haciéndolo, en el desarrollo de competencias, entre las que las digitales son una de las que mejor encajan en este nuevo perfil del ciudadano del siglo XXI.

La búsqueda del desarrollo digital del ciudadano se orienta a la potenciación, adquisición y formación de un conjunto de habilidades, conocimientos y actitudes digitales. Sin embargo, a esta perspectiva hay que añadir que el ciudadano debe saber actuar en situaciones problemáticas y ser capaz de encontrar soluciones a las mismas.

En este sentido, el Informe Horizonte de la Educación Superior 2020 (Brown et al., 2020) incide en que la Competencia Digital no es sólo en el sentido de que el ser humano sea competente en el uso de las diferentes tecnologías, sino que sea consciente de cómo éstas afectan al mundo digital, cómo integrar su uso y resolver posibles problemas.

Después de consultar diferentes definiciones de competencia digital, Gutiérrez \& Colmenero (2014, p. 121) construyó una explicación de lo que se puede entender como competencia digital: "valores, creencias, conocimientos, habilidades y actitudes para el uso adecuado de las tecnologías, incluyendo ordenadores, software e Internet, que permiten y posibilitan la búsqueda, el acceso, la organización y el uso de la información para construir conocimiento".

En las dos últimas décadas se han diseñado diferentes modelos que estructuran las competencias digitales a trabajar de diferentes maneras, bien desde un punto de vista general, dirigido al ciudadano, bien desde un punto de vista más específico, dirigido al profesorado universitario.

Se han consultado algunos de los modelos de autores como Mas Torelló (2011), Tejada Fernández (2009), Carrera Farrán y Coiduras Rodríguez (2012), Durán et al. (2016) y Ferrari (2013), con su Digcomp, un marco de referencia para desarrollar y comprender la competencia digital en Europa, que ha sido mejorado por el Intef (Instituto Nacional de Tecnologías Educativas y de Formación del Profesorado), en 2017, cuyas propuestas se dirigen principalmente al ámbito de la educación.

Junto a ello, se han desarrollado otras propuestas sobre el marco de la competencia digital del ciudadano, de manera que se ha ampliado a ámbitos como el de los empresarios, las 
organizaciones educativas, las políticas educativas, los consumidores y los estudiantes universitarios.

El Programa dentro del cual se encuentra el curso de Capacitación de Formadores en la Competencia Digital de Comunicación y Colaboración comprende las siguientes áreas competenciales, desarrolladas a partir del marco Digcomp 2.1 (competencia digital para los ciudadanos):

a) $\mathbf{1}^{\text {a }}$ actividad. Competencia de la Información y alfabetización informacional: Identificar, localizar, obtener, almacenar, organizar y analizar información digital, datos y contenidos digitales, evaluando su finalidad y relevancia para las tareas habituales;

b) $\mathbf{2}^{\mathrm{a}}$ actividad. Competencia de la Comunicación y colaboración: Comunicar en entornos digitales, compartir recursos a través de herramientas en línea, conectar y colaborar con otros a través de herramientas digitales, interactuar y participar en comunidades y redes; conciencia intercultural;

c) $3^{\text {a }}$ actividad. Competencia de la Creación de contenidos digitales: Crear y editar contenidos digitales nuevos, integrar y reelaborar conocimientos y contenidos previos, realizar producciones artísticas, contenidos multimedia y programación informática, saber aplicar los derechos de propiedad intelectual y las licencias de uso;

d) $4^{\text {a }}$ actividad. Competencia de la Seguridad: Protección de información y datos personales, protección de la identidad digital, protección de los contenidos digitales, medidas de seguridad y uso responsable y seguro de la tecnología;

e) $\mathbf{5}^{\mathbf{a}}$ actividad. Competencia de la Resolución de problemas: Identificar necesidades de uso de recursos digitales, tomar decisiones informadas sobre las herramientas digitales más apropiadas según el propósito o la necesidad, resolver problemas conceptuales a través de medios digitales, usar las tecnologías de forma creativa, resolver problemas técnicos, actualizar su propia competencia y la de otros.

Otra característica citada por esos autores está en la estrategia pedagógica, que debe ser diferenciada, pues hay peculiaridades en la construcción del conocimiento entre alumnos y profesores. Esta diferencia no está en el factor de la clase o en las actividades del aula, sino en la forma de demostración de los contenidos, como dice Santos (2020), dada la cantidad de medios disponibles. Este cambio, en el modo de transmisión de la información ha mejorado cada vez más con el uso de las TIC, aportando el papel del alumno no sólo como agente observador del proceso de enseñanza, sino también como miembro activo en este proceso.

El progreso tecnológico de las últimas décadas ha permitido un nuevo impulso, favoreciendo el creciente aumento y la democratización del acceso a la educación, poniéndose de manifiesto la importancia de la actuación de las universidades tras su adhesión al proceso educativo en cuestión.

\section{METODOLOGÍA: INFORME DE EXPERIENCIA}

A partir de los requerimientos de la convocatoria INTERCOONECTA se planteó el Programa de capacitación, fijando su alcance a la totalidad de los países prioritarios de la cooperación 
española en la región de América Latina y el Caribe y a través de este Plan, se seleccionaron los ODS de la Agenda 2030, las metas prioritarias y las líneas de acción de la Cooperación española vinculados a las competencias digitales que constituyen el eje central de la capacitación.

La formación en competencias digitales, bajo un mismo marco de referencia tomando en base el modelo europeo, pero adaptado a la realidad de América Latina, se considera un medio apropiado para contribuir al fortalecimiento e integración de la región.

Se presentan algunos conceptos introductorios, así como la metodología completa del curso y los principales retos y conclusiones obtenidos tras la experiencia transversal e interdisciplinar.

Para los cursos creados para los países latinoamericanos, se diseñaron metodologías de enseñanza híbrida, en las que habría semanas presenciales para enseñar las primeras nociones de las competencias digitales y luego pasar a la modalidad virtual. A medida que la pandemia avanzaba y se comprendía que había que sustituir los modelos de enseñanza, se desarrolló un nuevo diseño de instrucción para los cursos.

Hay diseños más didácticos, en los que los profesores guían los estudios, las lecturas, responden a las preguntas sobre los contenidos y gestionan la agenda del sistema. Cada alumno realiza sus propias tareas, dando cuenta de actividades casi siempre individualizadas. Esto es ODL. Los estudiantes aprenden y se gradúan. Pero preferimos invertir en más comunicación en la cibercultura y, para ello, insistimos en ONLINE. Por lo tanto, no es la materialidad de la red digital lo que garantiza la educación en línea. Lo que lo garantiza es el currículo que forjamos en la mediación interactiva e hipertextual de la comunicación y la producción de conocimiento en red (Santos, 2020, p. 9).

El programa de capacitación en competencias digitales compuesto por cinco cursos, basados en el Marco Europeo de Competencias Digitales (DIGCOMP), y adaptado a América Latina, destinado a personas seleccionadas en base al cumplimiento de requisitos especificados en el perfil de los postulantes, establecidos en la propuesta, consideró en sus objetivos fortalecer y movilizar las competencias digitales adquiridas durante la capacitación con el complemento de otras actividades formativas en línea.

El curso estaba previsto que funcionara de forma híbrida, donde las primeras nociones se impartirían de forma presencial y el seguimiento posterior de forma virtual, pero debido a la pandemia del nuevo coronavirus, el proyecto se modificó para trabajar totalmente a distancia.

Todos los participantes del curso de Capacitación de Formadores en la Competencia Digital de Comunicación y Colaboración, pertenecen a las administraciones públicas de países de América Latina y el Caribe (tales como Argentina, Cuba, Uruguay, Panamá, Bolivia y Perú) que tienen diferentes estructuras de apoyo para acceder a los medios de conocimiento.

Los participantes accedieron a la capacitación, luego de postularse a una convocatoria, cumplir los requerimientos de la misma y ser seleccionados en un proceso de evaluación de los antecedentes presentados, que requieren entre otros, acreditar ser empleados de la administración pública, estar avalados por su superior y presentar una propuesta de proyecto a desarrollar durante la capacitación, en caso de ser elegidos.

Esta contextualización es importante para entender incluso las dificultades que deben afrontar los docentes, cuando se trata de capacitar a los participantes en competencias digitales 
básicas de acceso a los espacios digitales, con retos y dificultades inherentes al uso de las TIC y las diferentes plataformas.

El Sistema de Información de Tendencias Educativas en América Latina (SITEAL, 2019) ha desarrollado numerosos estudios hasta la actualidad realizados con el objetivo de describir cómo se concibe lo educativo en las políticas públicas de inclusión de las TIC y cuáles son las acciones que se implementan con relación al ámbito pedagógico, la gestión, la infraestructura y conectividad. Todo ello, en pro de la creación de políticas públicas de alto impacto social que atiendan la inclusión social y educativa. Estos estudios han destacado varias temáticas como retos en materia de iniciativas educativas digitales: la integración curricular de las TIC, la presencia y gestión de las TIC en las escuelas, el desarrollo profesional docente, la brecha de género y las TIC.

Las clases se impartieron a través de la plataforma Microsoft Teams, para el desarrollo de las sesiones sincrónicas, así como la grabación para su posterior consulta por parte de los participantes; el Entorno Virtual de Enseñanza y Aprendizaje fue la plataforma Moodle de la institución AECID como podemos ver en la Ilustración 1.

\section{Ilustración 1 - Entorno virtual de aprendizaje del curso}
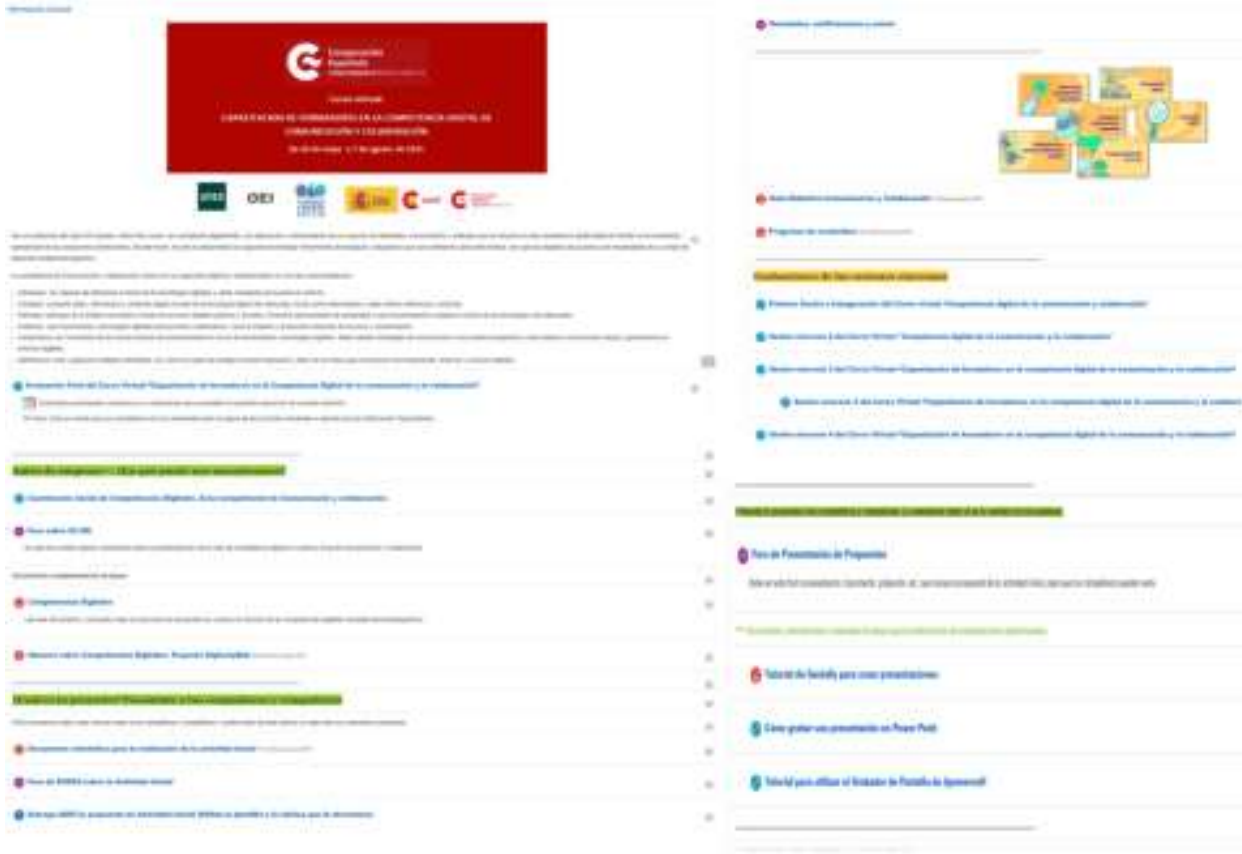

Nota. AECID (2021).

La metodología ABP, antes mencionada, fue originalmente proyectada para ser transversal al curso, para que el alumno, dentro de la competencia vigente, pudiera aplicar lo aprendido a la realidad de sus problemáticas planteadas, para poder resolverlos a partir de los conocimientos aprendidos, con la tutela del equipo de profesores.

La tutorización virtual de la formación se realizó principalmente a través del correo electrónico y foros habilitados a tal efecto; en estas actividades se abordaron los aspectos más 
relevantes del curso, permitiendo además la interacción entre docentes y participantes, el intercambio de experiencias e ideas y la resolución de dudas.

Al final del curso, cada participante entrega un proyecto sobre esa competencia, resolviendo algún problema relacionado con ella en su ámbito laboral y vinculado directamente a la institución pública donde labora.

Por otro lado, las acciones con resultados positivos fueron el intercambio de experiencias de los estudiantes, fomentado por la curiosidad de conocer la realidad entre países vecinos, en la esfera pública. Este hecho se debió a la comprensión de la reutilización de ciertas acciones en las obras que se estaban construyendo o incluso soluciones para acciones que no se vieron como un problema hasta un determinado momento.

Esto fue importante, incluso para el desarrollo de las clases como de las actividades del curso. La calidad y la velocidad de la conexión afectaban directamente a las clases presenciales. Estos factores combinados hicieron que la estrategia de construcción del curso tuviera una estructura de asignación de material en formatos ligeros, como los textos, y con el menor tamaño posible, dividiendo el material en varios archivos para facilitar el acceso de todos.

\section{CONCLUSIÓN}

Al observar el curso y los puntos principales del documento europeo de educación digital, podemos afirmar que los Los contenidos abordados favorecieron las acciones necesarias para resolver los temas.

Las competencias digitales ayudarán a los profesionales, sea profesor, gestor, investigador, a convertirse en ciudadano del siglo XXI competente digitalmente, pero también a ser un mejor profesional en su trabajo. Además, se pretende que se conviertan en formadores, ya sean usuarios, compañeros, colectivos cercanos, alumnos, entre otros.

El entrenamiento a escala permite llegar a diferentes puntos de los países y regiones de América Latina y el Caribe haciendo posible el desarrollo de la ciudadanía, a través de diferentes colectivos, proyectos sociales, centros de formación, centros culturales, centros educativos.

La experiencia obtenida en esta capacitación indica que se debe tener una mirada distinta en el diseño de instancias de formación cuando la intención no se reduce a la transmisión de información, sino a la resolución de problemas a escala institucional, que involucran a compañeros de trabajo y ciudadanos en general.

Como se puede observar, leyendo los objetivos de cada actividad formativa -área competencial, los conocimientos alcanzados por el participante en esta experiencia formativa conseguirán un perfil muy completo en cuanto a la competencia digital, siendo capaz de desenvolverse en el siglo XXI, inscripto en la era digital.

Es fundamental garantizar la mejora de las competencias digitales para la resolución de los desafíos, pero la amplia gama de áreas de competencia y la especificidad de las tecnologías hace que la gran acción es siempre el intercambio de los conocimientos obtenidos, lo que hace que diferentes acciones sean observadas por diferentes puntos de vista, dando la posibilidad de encontrar soluciones que implican diferentes competencias. 
Vale la pena mencionar que, aunque una persona sea experta en una competencia como comunicación y colaboración tiene que desarrollar la comprensión más cercana de las subcompetencias; es decir , interactuar a través de las tecnologías digitales y saber manejarlas de acuerdo al contexto. Compartir datos, información y contenido digital a través de la tecnología digital más adecuada. Participar en el ámbito comunitario a través de servicios digitales. Colaborar por medio de herramientas y tecnologías digitales más allá de la creación y producción de recursos digitales. Comportarse y ser consciente de las normas básicas de comportamiento en el uso de tecnologías digitales. Identificarse, crear y gestionar múltiples identidades, así como ser capaz de proteger la propia reputación y lidiar con los datos que se producen con herramientas, entornos y servicios digitales.

Así, en todos los ámbitos de la vida "Lo digital" está cada vez más presente alcanzando a los ámbitos educativo, laboral, social, económico, salud, investigación, gestión, etc. Por este motivo, es cada vez es más necesario que las personas adquieran el dominio en competencias digitales, siendo esta una preocupación a nivel internacional, la necesidad de formar a las personas como ciudadanos y ciudadanas del siglo XXI, esto fue posible lograr con este curso, que sin duda será un gran catalizador para el movimiento por el desarrollo de competencias digitales en América Latina y el Caribe.

\section{REFERENCIAS}

Aecid (2021). Cooperación Española de Conocimiento Intercoonecta. Recuperado de https://intercoonectaaulavirtual.aecid.es/login/index.php

Brown, M., McCormack M., Reeves. J., Brooks, C., Grajek, S., Alexander, B., Bali, M. Bulger, S., Dark, S., Engelbert, N., Gannon, K., Gauthier, A., Gibson, D., Gibson, R., Lundin, B., Veletsianos, G., \& Weber, N. (2020). EDUCAUSE Horizon Report, Teaching and Learning Edition, Louisville, CO. Recuperado de https://library.educause.edu/-/media/files/library/2020/3/2020_horizon_report_pdf.pdf

Carrera Farrán, F. X., \& Coiduras Rodríguez, J. L. (2012). Identificación de la competencia digital del profesor universitario: un estudio exploratorio en el ámbito de las ciencias sociales. REDU: Revista de Docencia Universitaria, 10(2), 273-298. doi: https://doi.org/10.4995/redu.2012.6108

Catapan, A. H. (2002). O presencial-atual e o presencial-virtual na EaD: construindo um plano de imanência. In Anais IX Congresso Internacional de Educação a Distância. São Paulo, Brasil: ABED. Recuperado de http://www.abed.org.br/congress02002/trabalhos/texto04.htm

Comisión Europea. (2006). Recomendação do Parlamento Europeu e do Conselho de 18 de Dezembro de 2006 sobre as competências essenciais para a aprendizagem ao longo da vida. Recuperado de https://eurlex.europa.eu/legal-content/PT/TXT/?uri=celex\%3A32006H0962

Comisión Europea. (2020). Comunicación de la comisión al parlamento europeo, al consejo, al comité económico y social europeo y al comité de las regiones. Plan de Acción de Educación Digital 2021-2027. Adaptar la educación y la formación a la era digital. Recuperado de https://eur-lex.europa.eu/legalcontent/ES/TXT/?uri=CELEX:52020DC0624 
Durán, M., Gutiérrez, I. \& Prendes, M. P. (2016). Análisis conceptual de modelos de competencia digital del profesorado universitario. RELATEC: Revista Latinoamericana de Tecnología Educativa, 15(1), 97-114. doi: https://doi.org/10.17398/1695-288X.15.1.97

Ferrari, A. (2013). Digcomp: A framework for developing and understanding digital competence in Europe. Luxembourg: Publications Office of the European Union. doi: http://dx.doi.org/10.2788/52966

Gutiérrez, R. C., \& Colmenero, M. J. R. (2014). La competencia digital en la formación de los futuros maestros: percepciones de los alumnos de los Grados de Maestro de la Facultad de Educación de Albacete. RELATEC: Revista Latinoamericana de Tecnología Educativa, 13(2) p. 119-133. doi: https://doi.org/10.17398/1695-288X.13.2.119

Lucas, M., \& Moreira, A. (2017). DigComp 2.1: quadro europeu de competência digital para cidadãos: com oito níveis de proficiência e exemplos de uso. Aveiro: UA. Recuperado de https://erte.dge.mec.pt/sites/default/files/Recursos/Estudos/digcomp2.1.pdf

Mas Torelló, O. (2011). El profesor universitario: sus competencias y formación. Profesorado, revista de currículum y formación del profesorado, 15(3), 195-211. Recuperado de http://www.ugr.es/local/recfpro/rev153COL1.pdf

Moreira, J. A., \& Schlemmer, E. (2020). Por um novo conceito e paradigma de educação digital onlife. Revista UFG, (20)26. dói: https://doi.org/10.5216/revufg.v20.63438

OCDE. (2019). Estrategias de competencias de la OCDE 2019. Competencias para construir un futuro mejor. Fundación Santillana. Madrid: OECD. Recuperado de https://www.oecd-ilibrary.org/education/estrategiade-competencias-de-la-ocde-2019_e3527cfb-es

Pimentel, M., \& Carvalho, F. S. P. (2020). Princípios da Educação Online: para sua aula não ficar massiva nem maçante! SBC Horizontes. Recuperado de http://horizontes.sbc.org.br/index.php/2020/05/23/principios-educacao-online/

Santos, E. (2020). EAD, palavra proibida. Educação online, pouca gente sabe o que é. Ensino remoto, o que temos para hoje. Mas qual é mesmo a diferença? Revista Docência e Cibercultura.

Setzer, V. W. (1999). Dado, informação, conhecimento e competência. DataGramaZero: Revista de Ciência da Informação. Recuperado de https://www.ime.usp.br/ vwsetzer/datagrama.html

Sistema de Información de Tendencias Educativas en América Latina (SITEAL]). (2019). Educación y TIC: Documentos de Eje. Buenos Aires: UNESCO. Recuperado de https://siteal.iiep.unesco.org/sites/default/files/sit_informe_pdfs/siteal_educacion_y_tic_20190607.pdf

Tejada Fernández, J. (2009). Competencias docentes. Profesorado. Revista de currrículum y formación del profesorado, 13(2), pp. 1-15. Recuperado de https://www.redalyc.org/pdf/567/56711798015.pdf

UNESCO. (2011). UNESCO ICT Competency Framework for Teachers. Paris, France: UNESCO. Recuperado de http://unesdoc.unesco.org/images/0021/002134/213475e.pdf

UNESCO. (2017a). Policy Papers Unesco: Sociedad digital: brechas y retos para la inclusión digital en América Latina y el Caribe. Montevideo, Uruguay: UNESCO. Recuperado de https://unesdoc.unesco.org/ark:/48223/pf0000262860?posInSet=3\&queryld=2d26415a-a44d-4b14-be4d2906ea1b3b5b

UNESCO. (2017b). Policy Papers Unesco: TIC, educación y desarrollo social en América Latina y el Caribe. Montevideo, Uruguay: UNESCO. Recuperado de https://unesdoc.unesco.org/ark:/48223/pf0000262862 
Uriarte Junior, F. A. (2008). Introduction to Knowledge Management. Jakarta, Indonesia: Asean Foundation.

Vuorikari, R., Punie, Y., Carretero Gomez, S. \& Van Den Brande, G. (2016). DigComp 2.0: The Digital Competence Framework for Citizens. Update Phase 1: the Conceptual Reference Model. Luxembourg (Luxembourg): Publications Office of the European Union. Recuperado de https://publications.jrc.ec.europa.eu/repository/handle/JRC101254 